\title{
Estructura de la red de la Organización Mundial de Comercio: desafíos y oportunidades para México
}

\author{
Fernando Lámbarry Vilchis *, Jorge Alejandro Silva \\ Rodríguez de San Miguel, Mara Maricela Trujillo Flores
}

Instituto Politécnico Nacional, CIIEMAD, México

Recibido el 22 de enero de 2017; aceptado el 22 de agosto de 2017

Disponible en Internet el: 14 de noviembre de 2018

\section{Resumen}

Los estudios en cuanto a la estructura de redes comerciales son recurrentes en configuraciones con un núcleo central y un bloque de países en la periferia, que fomentan inequitativos y desiguales intercambios. Consecuente con esto, el objetivo de este estudio es analizar estructuralmente la centralidad de la red económica de México en el marco de la Organización Mundial de Comercio y con ello identificar los desafíos y oportunidades para el país. El método de investigación implicó calcular métricas de centralidad fundamentadas en la teoría de redes. Se concluye que la red tiene una estructura estratificada, lo que desafía a México para replantear sus estrategias de inserción comercial internacional en América Latina y Asia Pacífico para el desarrollo y modernización de su economía.

Código JEL: F02, F10, F15.

Palabras clave: Análisis estructural de redes; Organización Mundial de Comercio; desafíos comerciales de México.

\footnotetext{
*Autor para correspondencia

Correo electrónico flambarry@ipn.mx (F. Lambarry Vilchis)

La revisión por pares es responsabilidad de la Universidad Nacional Autónoma de México.
} 


\begin{abstract}
The studies on the structure of commercial networks are recurrent in configurations with a central nucleus and a block of countries in the periphery that foment inequitable and unequal exchanges. Consistent with this the objective of this study is to analyze structurally the centrality of the economic network of Mexico in the World Trade Organization and with it identify the opportunities and challenges for the country. The research method involve calculates centrality metrics based on network theory. It is concluded that the network has a stratified structure what challenges Mexico to replant its strategies of commercial insertion in Latin America and Asia Pacific for the development and the modernization of its economy.
\end{abstract}

JEL code: $\mathrm{F} 02, \mathrm{~F} 10, \mathrm{~F} 15$.

Keywords: Structural Analysis of Networks; World Trade Organization; Trade Challenges of Mexico.

\title{
Introducción
}

La concepción de una estructura conformada por un centro y una periferia es un paradigma en el estudio del sistema mundial ejemplo de ello es el modelo estratificado de Wallerstein (1979) de núcleo, semi periferia y periferia, en el que se ha examinado la jerarquía internacional y el intercambio comercial dentro y entre los estratos del sistema, que, si bien no existe consenso sobre su validez general, los estudios reiteran la existencia de estratos, aunque difieren de su número. Es en el núcleo central, cohesivo, con alta densidad de interrelaciones donde residen los países desarrollados y en las periferias débilmente conectadas entre sí los menos desarrollados (Borgatti y Everett, 1999; Wallerstein, 1979) fomentando con esta configuración, asimetrías regionales que benefician más al centro que al perímetro (Prebisch, 1964;1950) con patrones de exportación de alta tecnología y productos procesados que circulan dentro del núcleo y hacia estratos inferiores, mientras que, la exportación de productos agrícolas y de materias primas de la periferia a los bloques superiores incluyendo al núcleo (Smith y White, 1992) no obstante, los países periféricos comercian más con el núcleo básico del que han sido dependiente que con otros países de la periferia que están geográficamente más cerca (Wallerstein, 1979; Smith y White, 1992). Consecuente con esto, desde la perspectiva teórica de redes se han aplicado métricas de centralidad para confirmar la existencia de esta configuración estructural (Nordlund, 2013; Smith y White, 1992). Es por ello que el objetivo de este estudio consistió en analizar la centralidad estructural de la red económica de las exportaciones e importaciones de mercancías de México en el marco de la Organización Mundial de Comercio, para identificar los desafíos y oportunidades para el país. Para ello, se empleó un método de investigación de corte cuantitativo que implicó estudiar el intercambio económico del país bajo tres atributos estructurales de la métrica de centralidad: cercanía, grado e intermediación (Cherven, 2013; Jackson, 2008; Newman, 2003; Freeman, 1977, 1979) y el coeficiente de cohesión (Watts y Strogatz, 1998). Los resultados avalan una configuración estratificada de la red, que advierte a los tomadores de decisiones gubernamentales una necesidad urgente de revisar y replantear las estrategias de inserción comercial internacional de México en América Latina y la región Asia Pacífico como prioritarias para el desarrollo y modernización de su economía. 


\section{México en la Organización Mundial del Comercio}

El crecimiento del comercio mundial se ha desacelerado significativamente desde el año 2012, el volumen de bienes y servicios ha crecido un poco más del 3\% al año, menos de la mitad de la tasa media de expansión durante las tres décadas anteriores (International Monetary Fund, 2016). Las razones de la desaceleración del comercio aún no se entienden claramente, sin embargo, algunas de ellas han sido la disminución en el ritmo de la liberalización comercial y el reciente aumento en las medidas proteccionistas (Evenett y Fritz 2015), pese al fuerte consenso entre los economistas de que una mayor integración comercial contribuiría al aumento de la productividad y al desarrollo de las economías, elevando el bienestar general (International Monetary Fund, 2016). Es por ello que, para favorecer a que el comercio fluya con la mayor libertad posible, la Organización Mundial de Comercio (OMC) lo ha fomentado a través de normas mundiales en sus 164 países miembros (Organización Mundial del Comercio, 2016a) que representan más del $90 \%$ de los países en el mundo incluidas las economías más grandes en cuanto al tamaño de su Producto Interno Bruto (PIB). China, Estados Unidos y Alemania son las naciones que lideran tanto las exportaciones como importaciones en términos del valor de su comercio internacional de mercancías en la OMC, mientras que México se encuentra en la posición 15 como exportador y en la 14 como importador (Organización Mundial del Comercio, 2016b), el país ostenta el lugar 15 en cuanto al tamaño de su economía (The World Bank, 2016), es el sexto receptor de flujos de Inversión Extranjera Directa más importante de las economías en desarrollo y de transición (United Nations Conference on Trade and Developmen, 2016). En cuanto al sector energético, México es el décimo país productor de petróleo crudo y el doceavo en gas natural a nivel mundial (Pemex, 2014), mientras que en su producción minera ocupa el lugar número 12 (Bancomext, sin año). En la facilidad para hacer negocios México ocupa la posición 38 de 189 países (Banco Mundial 2016) y en el ranking de competitividad está en el número 57 de 140 naciones (World Economic Forum, 2016), que revelan el potencial para que el país se posicione mejor en la estructura de comercio exterior no obstante, resultan insuficientes para una eventual transición de la periferia al centro, lo que además se reafirma en su balanza comercial de mercancías al importar 411, 581 millones de dólares y exportar sólo 397,506 millones de dólares con un balance negativo (World Trade Organisation, 2015a) o bien, por su recurrente promedio de crecimiento en los últimos diez años menor en sus importaciones con un $6 \%$ respecto de un $6.5 \%$ de las exportaciones del país (Secretaría de Economía, 2015). Aunque si bien, todo ello en gran medida puede explicarse por la alta dependencia comercial de México con los Estados Unidos al concentrar el 78.9\%\% del total de sus mercancías exportadas, seguido, aunque distantemente en un 5.2\% a la Unión Europea (World Trade Organisation, 2015a). Entre los principales productos que les exporta México se encuentran el petróleo crudo con el $11 \%$ y vehículos automotores de pasajeros con el 9\% del total de sus exportaciones (Organisation for Economic Cooperation and Development y World Trade Organization, 2015). Mientras que, de forma similar para el caso de las mercancías importadas por el país provienen en su gran mayoría con un 49.3\% de los Estados Unidos, un $16.1 \%$ de China y un 11.3\% de la Unión Europea (World Trade Organisation, 2015a), sobresale la importación de partes y equipo de telecomunicaciones y los productos del petróleo ambos con un 7\% del total de productos importados (Organisation for Economic Cooperation and Development y World Trade Organization, 2015). Es por ello, que esta evidente relación de dependencia con la principal potencia del mundo ha traído como una probable consecuencia 
que el comercio con otros países o regiones sea menor (tabla 1) además de impedirle explorar nuevos mercados a pesar de que México es el segundo país con más tratados de libre comercio en el mundo con 234 bilaterales por 122 multilaterales (Secretaría de Relaciones Exteriores, 2016) y un fuerte potencial para combinar la diversificación de sus destinos exportadores lo que le permitiría eventualmente transitar en la estructura de la economía mundial hacia los países con niveles altos de ingresos (Foxley, 2012).

Tabla 1 Exportaciones e importaciones de México por regiones

\begin{tabular}{lllll}
\hline Regiones & $\begin{array}{l}\text { Exportaciones } \\
\text { (millones de dólares) }\end{array}$ & $\begin{array}{c}\text { Porcentaje } \\
\text { Exportaciones }\end{array}$ & $\begin{array}{l}\text { Importaciones } \\
\text { (millones de dólares) }\end{array}$ & Porcentaje \\
\hline Norteamérica & 319333.6 & 86.26 & 196750.0 & 54.29 \\
Unión Europea & 18440.0 & 4.98 & 43722.4 & 12.06 \\
Asia & 12278.30 & 3.31 & 110201.40 & 30.40 \\
Sudamérica & 15084.3 & 4.07 & 9601.2 & 2.64 \\
Centro América & 5037.1 & 1.36 & 2118.7 & 0.584 \\
Total: & 370173.30 & $100 \%$ & 362393.70 & $100 \%$ \\
\hline
\end{tabular}

Fuente: elaboración propia a partir de: Secretaría de Economía, (2015) y World Trade Organisation, (2015a).

\section{Base teórica: estructura centro-periferia}

La explicación alternativa bajo los preceptos teóricos de dependencia para entender la dinámica del desarrollo global y las relaciones entre los países desarrollados y en desarrollo, ha recibido considerable atención de los economistas en todo el mundo, compartiendo la presunción de un sistema mundial con una estructura estratificada (Steiber, 1979) por un núcleo, periferia y semi periferia (Wallerstein, 1979) con relaciones cada vez más interdependientes (Kali y Reyes, 2010) y complejas, que para su entendimiento el estudio tradicional de los flujos comerciales bilaterales está siendo reemplazado por nuevos métodos de análisis fundamentados en la teoría de redes. En la literatura internacional sobresalen tres redes clásicas: las aleatorias (Erdös y Rényi, 1960), las de escala libre (Reka y Barbási, 2002) y las de pequeño mundo (Watts y Strogatz, 1998). En ellas se analizan varios aspectos de sus propiedades estructurales y topológicas en sus formas no dirigida, dirigida, binaria o ponderada. Bajo esta perspectiva de redes existen estudios que analizan las relaciones comerciales internacionales (Wlather, 2015; De Benedictis y Tajoli, 2011; Serrano, Boguñá y Vespignani, 2007). Algunos de ellos utilizan métricas de red para describir su estructura y complejidad (Rombach, Porter, Fowler y Mucha, 2014, Barigozzi, Fagiolo y Mangioni, 2011; Bhattacharya, Mukherjee, Sar"amaki, Kaski y Manna 2008; Mahutga, 2006; Garlaschelli y Loffredo, 2005; Serrano y Boguñá, 2003) y, al hacerlo, intentan medir hasta qué punto la economía global se ha integrado o ha evolucionado 
en el tiempo (Reyes, Schiavo y Fagiolo, 2008; Kim y Shin, 2002; Fujita, Krugman y Venables, 1999), o bien, sólo en ciertas regiones y/o sectores específicos (Lámbarry, 2016; Amador y Cabral, 2016; Cerina, Zhu, Chessa y Riccabon, 2015; Ferrarini, 2013). Otros estudios consideran cómo los países individuales están condicionados por su posición dentro de la red y/o su nivel de centralidad (Prell, Sun, Feng y Myroniuk, 2015; Mahutga, 2006), distinguiendo entre los países que se sitúan en el núcleo frente a los de su periferia. En el núcleo se encuentran las naciones económicamente diversificadas, ricas, poderosas y relativamente independientes del control externo (Chirot, 1977). En el otro extremo las naciones periféricas, resultado de la explotación histórica y su alta dependencia de los países del núcleo se encuentran en el subdesarrollo y permanente pobreza (Richardson, 1986; Beckford, 1973).

Es en el núcleo central, innovador, cohesivo, con alta densidad de interrelaciones donde residen los países generadores y propagadores de progreso técnico y líderes de la especialización productiva mundial y en las periferias supeditadas a los primeros en cuanto a dependencia tecnológica y posicionamiento productivo internacional débilmente conectadas entre sí, los menos desarrollados (Borgatti y Everett, 1999; Friedman, 1973; Prebisch, 1950). Un país puede ser llamado centro de la economía mundial si desempeña un papel dominante en el comercio internacional y el movimiento del capital normalmente es a partir de él a otros países. Por el contrario, un país es considerado en la periferia si desempeña un papel secundario o pasivo en el comercio mundial lo que lo hace altamente dependiente del núcleo (Cárdenas, 2014; Meier y Baldwin, 1957). Son estos países de los bloques no centrales los que comercian más con el núcleo del que han sido históricamente dependiente, más que con otros países de la periferia aun cuando están geográficamente más cerca (Wallerstein, 1979; Smith y White, 1992) dibujando un patrón de intercambio desigual con flujos asimétricos de productos menos procesados desde la periferia a un cambio de costosos productos terminados desde el núcleo (Rosales, 2015) lo que beneficia más al centro independientemente de donde se produzcan (Prebisch, 1950). Es por ello que, mediante el cálculo de la estructura núcleo-periferia de una red, se intenta determinar qué nodos son parte de un núcleo densamente conectado y cuáles forman parte de una periferia poco conectada. Una de las metodologías más populares para investigar la estructura núcleo-periferia fue la propuesta por Borgatti y Everett (1999), aunque más recientemente a través de software de análisis de redes es posible identificarla y con ello, en el tema de la estructura comercial, validar el papel de las políticas económicas, industriales y de exportación de los países que podrían conducir a cambios y alterar la posición estructural de un país en el ámbito internacional económico (Smith y White, 1992) o bien, si el escenario mundial está generando nuevas relaciones asimétricas en que los centros podrán consolidar su hegemonía y acrecentar las distancias económicas con las regiones periféricas (Di Filippo, 1988; 1997).

\section{Método de investigación}

Esta investigación de corte cuantitativo y transversal planteó el objetivo de analizar estructuralmente la centralidad de México en la red económica de sus importaciones y exportaciones en el marco de la Organización Mundial de Comercio para conocer su estructura y funcionamiento y con ello identificar los desafíos y oportunidades para el país. 
El estudio se realizó a través del método empleado por Lámbarry (2016) y fundamentado en la teoría de redes, particularmente en el análisis de Freeman (1979) de la métrica de centralidad para nodos en sus tres propiedades estructurales, adecuadas al contexto comercial sin perder el sentido de su dimensionalidad: 1. Cercanía. 2. Grado y 3. Intermediación. Adicionalmente, se consideraron dos métricas globales: 1. Índice de centralidad del grafo de Freeman (1979) y, 2. Coeficiente de cohesión de Watts y Strogatz (1998). Para determinar el grado de interconexión de los países en la red comercial.

El estudio implicó dos fases de análisis de centralidad para dos redes comerciales, la primera para la red de la Organización Mundial de Comercio y enfocándolo particularmente hacia México y, una segunda fase para la subred identificada y conformada por los socios directos con el que México comercia mercancías.

\section{Métricas de centralidad: nodos y grafos}

Se define como grafo a un conjunto de puntos o nodos no ordenado $\left(p_{i}, p_{j}\right)$ donde cada uno es alcanzable desde el otro, si existe una secuencia de una o más aristas, $\left(p_{i}, p_{a}\right),\left(p_{a^{\prime}} p_{b}\right),\left(p_{b^{\prime}}\right.$, $\left.p_{c}\right) \ldots\left(p_{z}, p_{j}\right)$, que comienza en $p_{i}$, y, posiblemente pase a través de puntos intermedios, $p_{a}, p_{b}$, $p_{c} \ldots p_{z}$, terminando en $p_{j}$ (Freeman, 1979).

La métrica de centralidad puede determinarse tanto para un punto como para un grafo por referencia a cualquiera de sus tres atributos estructurales (Freeman, 1979): grado (degree), intermediación (betweenness) y cercanía (closeness), oscila entre dos valores: uno al existir pocos actores centrales que concentran los enlaces y el mayor número de relaciones, mientras que un valor de cero significa que todos los nodos están unidos entre sí; ningún actor es más central que otro.

Para la interpretación de las tres métricas nodales de centralidad que fueron empleadas en este estudio, se consideró:

La centralidad de un nodo en términos del grado de un punto $p_{i}$, es simplemente el número de puntos $p_{j}(i \neq j)$ que son adyacentes a él (Freeman, 1979). Esta medida es un indicador sobre la actividad potencial de comunicación del punto, se calcula como el grado o el número de adyacencias para el punto $p_{k}$ (Nieminen, 1974), (ecuación 1).

$$
C_{D}\left(p_{k}\right)=\sum_{i=1}^{n} a\left(p_{i}, p_{k}\right) \ldots
$$

La centralidad basada en la propiedad estructural de intermediación, considera central al punto en la medida de su potencial para controlar la comunicación (Freeman, 1979), (ecuación 2).

$$
C_{B}\left(p_{k}\right)=\sum_{i<}^{n} \sum_{j}^{n} b_{i j}\left(p_{k}\right) \ldots(2)
$$

En cuanto a la centralidad de cercanía de un punto, lo considera central en la medida en que puede evitar el potencial control de los otros. Es una medida de descentralidad o centralidad inversa, crece a medida en que los puntos están separados lo que en este sentido significa cercanía. La independencia de un punto está determinada por su cercanía a todos los demás puntos del grafo (Freeman, 1979). Beauchamp (1965) propone calcularlo con la ecuación: 


$$
C^{\prime}{ }_{c}\left(p_{k}\right)=\frac{n-1}{\sum_{i=1}^{n} d\left(p_{i}, p_{k}\right)} \ldots
$$

Mientras que para la interpretación de las métricas de la centralidad del grafo se consideró:

El índice de centralidad (ecuación 4) de Freeman (1977), el cual se determina a partir de alguna de las tres diferentes medidas de la centralidad de un punto. El grafo resulta compacto en la medida en que las distancias entre los pares de puntos que lo conforman son pequeñas.

$$
C_{x}=\frac{\sum_{i=1}^{n}\left[C_{x}\left(p^{*}\right)-C_{x}\left(p_{i}\right)\right]}{\max \sum_{i=1}^{n}\left[C_{x}\left(p^{*}\right)-C_{x}\left(p_{i}\right)\right]} \ldots
$$

El coeficiente de cohesión, agrupamiento o transitividad $C$, de Watts y Strogatz (1998), que cuantifica qué tanto está agrupado el nodo (o interconectado) con sus vecinos (ecuación 5):

$$
C_{i}=\frac{a_{i}}{k_{i}\left(k_{i}-1\right) / 2} \ldots
$$

Los valores de estos coeficientes oscilan entre un valor de cero (sin conectividad) y uno (conectividad total).

\section{Recolección de datos}

Para el análisis de redes, se recopiló información de la Base de Datos Estadística y el Perfil comercial de la Organización Mundial de Comercio sobre el intercambio económico comercial entre los países socios vigentes al año 2015 de: http://stat.wto.org/CountryProfile/ WSDBCountryPFHome.aspx ?Language=E, lo cual implicó construir una matriz con una estructura de país origen, tipo de relación (importación o exportación), país destino y valor de la relación (expresado en porcentaje de importación y exportación de acuerdo a los países de origeno destino principales) lo que dio por resultado una matriz con un tamaño de 1,528 registros, necesaria para el cálculo de los coeficientes de centralidad y cohesión en el software de análisis de redes sociales Cytoscape ver 3.3.0, (anexo tabla A1). El tratamiento de los datos no implicó un proceso de homologación ni depuración por la correcta estandarización de la base de datos de la OMC.

\section{Análisis y resultados}

La red de económica comercial generada por los intercambios de importaciones y exportaciones de mercancías entre los miembros de la Organización Mundial de Comercio presenta como parámetros generales un total de 186 nodos, que representa la totalidad de los países que mantienen alguna relación comercial (anexo figura 1). De ellos, es la Unión Europea el conjunto de países con la mayor actividad potencial de comercialización en la red al tener la métrica de grado más alta con 287, seguido por China con 221 y Estados Unidos con 204, muy distantes de Japón quien se encuentra en cuarto lugar con un valor de 80 . El coeficiente de cohesión de la red representa el valor que indica la existencia de un 52\% de interconexión 
entre los socios. En este sentido, Honduras, Hungría y Bulgaria tienen un coeficiente de uno, le siguen Uruguay, Luxemburgo e Irlanda con 0.9333. No obstante, resalta que la Unión Europea, China y Estados Unidos son los países con los coeficientes de cohesión más bajos, con valores por debajo de 0.1 , mientras que 24 naciones presentan coeficientes con valores de cero.

El índice de centralidad de 0.743 evidencia que la red es compacta y en general que existe una fuerte cercanía comercial entre todos los países en el marco de la Organización Mundial del Comercio. Esta alta centralidad revela también la existencia de al menos un miembro en la red económica que por su posición nuclear domina la de los demás (Unión Europea, China y Estados Unidos). Una de las grandes desventajas de esta estructura es una integración poco efectiva, en la que eventualmente si el país central se desincorpora, la conexión entre el resto de los países se desestabiliza lo que pondría en riesgo la economía y relaciones comerciales multilaterales del bloque. Aunque a nivel nodo estos valores se asocian con la independencia del país respecto de otros, en este sentido, la Unión Europea al tener el coeficiente de mayor valor con 0.814 representa el grupo de naciones con la mayor independencia en cuanto a sus intercambios comerciales, seguido por China con un coeficiente de 0.793 y Estados Unidos con 0.702. Por lo que son estos países los que conforman el núcleo de la red coincidiendo con su mayor peso económico en términos del Producto Interno Bruto con relación de los demás socios comerciales.

En esta misma tendencia, nuevamente es la Unión Europea quien presenta la métrica de intermediación más alta con un valor de 0.319 , le sigue China con 0.287 y Estados Unidos con 0.163 , esto representa el significativo potencial de estos países para controlar el intercambio de la actividad comercial que detentan una marcada diferencia del resto de los miembros de la OMC que están por debajo de valores de 0.04 .

Desde esta perspectiva, México en la red de la Organización Mundial del Comercio (anexo tabla A2) tiene un coeficiente de cohesión de 0.470 , ligeramente por debajo del coeficiente promedio de la red $(0.522)$ por lo que el país tiene una interconexión por debajo de la media con otros países del $47 \%$, esto indica un claro desaprovechamiento de su potencial para establecer intercambios comerciales con los demás socios de la OMC limitándose a un número reducido de ellos (países centrales) o bien a un bajo nivel de exportaciones e importaciones (socios de la periferia).

La métrica de cercanía del país es de 0.479 que indica que el país es sensiblemente independiente respecto de los demás al estar por arriba de la media de 0.450 , aunque si bien

como referencia dista del valor más alto de 0.814 , pese a estas métricas, el coeficiente de intermediación de México es aún más bajo que el valor promedio de la red de 0.007 por 0.001 del país, de tal forma que su potencial para controlar el intercambio comercial es inexistente. En definitiva, salvo este último coeficiente que se encuentra distante de la media lo que es negativo en términos de centralidad, las otras dos métricas se encuentran próximos a los promedios pero distantes de los países centrales, lo que confirma una posición periférica del país que se explica en primera instancia por su nula generación de progreso técnico pero también por una escasa diversificación e integración interna de sus estructuras productivas y exportadoras.

Ahora bien, al seleccionar la subred que forma México se identifican a 19 nodos o países que la conforman (anexo) y en un primer análisis comparativo de los valores promedios de las métricas globales de centralidad respecto de las de la red de la Organización Mundial del Comercio, se observa en la subred una menor cohesión con un coeficiente de 0.507 contra 0.522 , una mayor cercanía de 0.523 por 0.45 de la red OMC y una mayor intermediación de 
0.043 contra 0.007 . Lo que indica que esta subred comercial tiene una menor interconexión entre los países y por lo tanto un menor flujo de intercambio de exportaciones e importaciones de mercancías, además, una mayor independencia comercial entre ellos y la inexistencia de un país que controle el comercio. Aunque en esta subred se confirma la misma estructura de la red de la OMC de países con los coeficientes más altos de cercanía, la Unión Europea con un valor de 0.814 , China con un coeficiente 0.793 y Estados Unidos con 0.702 , si bien no significativos de los del resto de los demás países del bloque, no obstante, esto señala que a pesar de existir una integración mercantil, estos países líderes tienen una alta independencia comercial por lo que no serían afectados en caso de interrumpirse las relaciones comerciales, salvo sensiblemente Honduras al tener el menor valor de cercanía (0.464) pero que no dista mucho del promedio de la red de 0.523 . Este es el mismo caso para México que con un coeficiente de cercanía de 0.479 se encuentra ligeramente por debajo del valor promedio.

Respecto a la métrica de intermediación, nuevamente se replica esta tendencia estratificada de países que conforman el núcleo de la subred al ser los mismos que lideran la métrica de cercanía: la Unión Europea con 0.319, China con 0.287 y Estados Unidos con 0.163, incluso con valores muy por arriba del resto de los demás países que promedian escasamente

0.043. Lo mismo ocurre para el coeficiente de grado, mientras que Japón se ubica en un cuarto lugar con un valor de 80 dista de los países líderes, Estados Unidos con un coeficiente de 204, de China con 221 y de la Unión Europea con 287, coeficiente que representa los países con los que mantienen intercambios comerciales de mercancías.

En el tema del coeficiente de cohesión, Honduras presenta el mayor de los valores toda vez que es de magnitud de uno y de igual forma, esta nación presenta también los valores más bajos de cercanía e intermediación, seguida por El Salvador con una cohesión de 0.982 quien comparte con Honduras los valores más bajos en los otros dos coeficientes de centralidad, que si bien indican una buena interconexión de estos países con los demás del bloque, también evidencian una alta dependencia y un bajo potencial en el control comercial en la subred. En contraste, la Unión Europea con un coeficiente de 0.071, China de 0.072 y Estados Unidos con 0.086 son los países con los valores más bajos de cohesión, incluso muy por debajo del promedio de 0.507. Esto revela su poco interés por una interconexión e integración efectiva comercial de la red, atribuible en gran medida por mantener su status quo asociado a su posición central y a la falta de políticas y acuerdos comerciales más equitativos que estimulen el desarrollo económico y comercial de los países.

Por otra parte, un análisis correlacional determinó que el coeficiente de cohesión presentó una correlación inversa negativa muy marcada de -0.728 con el de cercanía y de -0.65 con el de intermediación, mientras que estos dos últimos coeficientes presentaron una correlación alta de 0.998. Lo que confirma que aquellos países con los valores más altos de cercanía e intermediación tienen los más bajos valores de cohesión y aquellas naciones con los valores más altos de cercanía tienen de igual forma los más altos de intermediación.

En suma, China, Estados Unidos y la Unión Europea son los países centrales de la subred de México que tienen los coeficientes más altos de centralidad estructural, con excepción del de cohesión, Japón, Brasil, República de Corea y Canadá son las economías con mayores posibilidades de eventualmente transitar al núcleo, sensiblemente más distante de ellos se encuentra México pero con mejores coeficientes estructurales de centralidad que el resto de los países centroamericanos y sudamericanos de la periferia (anexo). 


\section{Conclusiones}

Una primera conclusión de este estudio derivado de las métricas de centralidad de la red económica de exportaciones e importaciones de la Organización Mundial de Comercio es la existencia de estructuras jerárquicas estratificadas, en coincidencia con los planteamientos de Smith y Wallerstein (1979), Smith y White (1992) y Nordlund (2013) en sus estudios del sistema mundial. La red presenta una configuración nuclear dominada por China, la Unión Europea y Estados Unidos que son los países con los coeficientes de mayor valor de cercanía, grado e intermediación, presumiblemente en correlación con el tamaño de sus economías y confirman desde estas métricas y el concepto centro-periferia que son generadores y propagadores de progreso técnico y rectores de la especialización productiva mundial.

Esta misma configuración estratificada se replica en la subred de México y sus socios comerciales directos, que apunta a una estructura centralizada poco efectiva que favorece a las economías del núcleo, con alta dependencia de los países periféricos con los centrales, una configuración en la que eventualmente si el país central se desincorporara el intercambio comercial entre el resto de los países se desestabilizaría poniendo en riesgo sus economías. Por lo tanto, estas naciones por su posición central dominan el intercambio comercial como lo sugieren Friedmann (1966) y Uzzi y Spiro (2005). Además, estos países del núcleo poseen bajos coeficientes de cohesión, que contrastan con los del resto de los demás países, revelando su poco interés por una interconexión e integración comercial efectiva de la red, atribuible entre otros factores, en gran medida por mantener su status quo asociado a su posición central en la red.

Aunque desde la perspectiva de la periferia, por la proximidad de los valores de los coeficientes de centralidad y cohesión de estos países se concluye que se configura una periferia equitativa y homogénea, no obstante, sus bajos coeficientes e inferiores al valor promedio resulta una estructura comercial ineficaz con poco alcance de estos países hacia su inserción en la economía mundial.

Los resultados del presente estudio confirman a México como un país ubicado en la periferia de la red de la OMC, por lo que se sugiere se debe impulsar su competitividad y desarrollo económico, una de las opciones para lograrlo es profundizar sus lazos comerciales con Centroamérica y Sudamérica, específicamente con los países con los que por su posición estructural conforman la periferia de la subred de la que forma parte, inicialmente con Colombia, Nicaragua y Belice quiénes poseen métricas similares de centralidad y que representarían una cadena de suministro para los productores mexicanos en la exportación de productos finales a la región Asia-Pacífico. Es necesario consolidar una integración comercial regional para el dinamismo futuro de la economía mexicana ya sea mediante un bloque regional exclusivamente asiático, uno del Asia-Pacífico, o uno centrado en China.

Una conclusión adicional que refuerza la anterior, radica en consolidar el mercado asiático que ha desplazado al latinoamericano (anexo), lo que se confirma desde la perspectiva de los altos coeficientes de centralidad de China, Japón y la República de Corea. Estos países representan el mercado potencial para una gran variedad de productos de consumo que la economía mexicana puede ofrecer en condiciones competitivas. No obstante, la amenaza creciente de la manufactura China que le hace perder a México presencia en los mercados globales, la perspectiva de un largo período de lento crecimiento de la Unión Europea y la alta vulnerabilidad de México por la dominante dependencia comercial con los Estados Unidos, es imperante una estrategia enfocada más a diversificar sus destinos y estructuras exportadoras. 
Derivado de lo anterior, se plantea una necesidad urgente de revisar y replantear las estrategias de inserción internacional de este país en América Latina y la región Asia Pacífico como prioritarias para el desarrollo y modernización de su economía.

Una recomendación a futuros trabajos en este tema es profundizar el análisis de la centralidad de la estructura por tipo de mercancía exportada e importada de la subred de México, para identificar el comercio basado en cadenas de valor productivas regionales y estratégicas al que deben enfocarse estos países para el desarrollo equitativo de sus economías, tarea que no se percibe fácil por los costos implicados por los potenciales cambios estructurales en el sistema económico.

\section{Referencias}

Amador, J. \& Cabral, S. (2017). Networks of Value-added Trade. The Work Economy, 40 (7), 1291-1313. https://doi. org/10.1111/twec.12469

Bancomext. (sin año). Macro Coyuntura Minería. Disponible en: http://www.bancomext.com/wp-content/uploads/2015/08/Minero_1er_informe_2015.pdf

Banco Mundial (2016). Doing Business. Disponible en: http://espanol.doingbusiness.org/rankings

Barigozzi, M., Fagiolo, G., Mangioni, G. (2011). Identifying the community structure of the international-tradmulti-network. Physica A: Statistical Mechanics and its Applications. 390(11), 2051-2066. https://doi.org/10.1016/j.physa.2011.02.004

Bhattacharya, K., G. Mukherjee, J. Sar"amaki, K. Kaski \& Manna, S. (2008). The International Trade Network: Weighted Network Analysis and Modeling. Journal of Statistical Mechanics: Theory and Experiment. A, 2: P02002.

Beauchamp, M.A. (1965). An improved index of centrality. Behavioral Science, 10,161-163. https://doi.org/10.1002/ bs.3830100205

Beckford, G. (1973). Review: Persistent Poverty: Underdevelopment in Plantation Economies of the Third World. The Economic Journal, 83(332), 1321-1324. DOI: 10.2307/2230878

Borgatti, S.P. \& Everett, M.G. (1999). Models of core-periphery structures. Social Networks, 21, 375- 395. DOI: 10.1016/S0378-8733(99)00019-2

Cárdenas, J. (2014). El poder económico mundial: Análisis de redes de interlocking directorates y variedades de capitalismo. Madrid: Centro de Investigaciones Sociológicas.

Cerina, F., Zhu, Z., Chessa, A., \& Riccaboni, M. (2015). World Input-Output Network. PLOS ONE, $10(7)$, e0134025. https://doi.org/10.1371/journal.pone.013402

Cherven, K. (2013). Network Graph Analysis and Visualization with Gephi. United Kindom: Packt Publishing.

Chirot, D. (1979). Review: Social Change in the Twentieth Century. American Journal of Sociology, 84(5), 1275-1278 http://www.jstor.org/stable/2778234

De Benedictis, L. \& Tajoli, L. (2011). TheWorld Trade Network. The World Economy, 8(34), 1417-1454. doi: 10.1111/j.1467-9701.2011.01360.x

Di Filippo, A. (1997). Multilateralismo y Regionalismo en la Integración de América Latina. Documentos de trabajo, No. 46, Santiago de Chile: Comisión Económica para América Latina y el Caribe. Disponible en: http://repositorio. cepal.org/bitstream/handle/11362/7367/S9700500_es.pdf?sequence =1\&isAllowed=y

Di Filippo, A. (1988). La visión centro-periferia hoy. Revista de la CEPAL. No. Extraordinario, 175-185. Disponible en: http://www.cepal.org/publicaciones/xml/1/19381/difil.htm

Evenett, J. S, \& Fritz, J. (2015). The Tide Turns? Trade Protectionism and Slowing Global Growth, Global Trade Alert, Centre for Economic Policy Research Press. Disponible en: http://voxeu.org/sites/default/files/file/GTA18_final.pdf 
Ferrarini, B. (2013). Vertical trade maps. Asian Economic Journal, 27(2), 105-123. doi: 10.1111/asej.12005

Foxley, A. (2012). La trampa del ingreso medio. El desafío de esta década para América Latina. Chile: CIEPLAN.

Disponible en: http://www.cieplan.org/media/publicaciones/archivos/308/Libro_Digital_La_trampa _del_ingreso_medio.pdf

Friedmann, J. (1973). Urbanization, planning and national development. Beverly Hills: Sage.

Freeman, L. (1979). Centrality in Social Networks Conceptual Clarification. Social Networks, 1, 215-239. https://doi. org/10.1016/0378-8733(78)90021-7

Freeman, L. (1977). A Set of Measures of Centrality Based on Betweenness. Sociometry, 40(1), 35-41. DOI: $10.2307 / 3033543$

Fujita, M, Krugman P. \& Venables, A. (1999). The Spatial Economy: Cities, Regions and International

Trade. Cambridge: The MIT Press. Disponible en: http://geografi.ums.ac.id/ebook/The_Spatial_Economy-Fujita_Krugman__Venables.pdf

Garlaschelli, D., \& Loffredo, M. (2005). Structure and evolution of the world trade network. Physica A, Statistical Mechanics and its Applications, 355(1), 138-144. https://doi.org/10.1016/j.physa.2005.02.075

International Monetary Fund (2016). World Economic Outlook (WEO). Subdued Demand: Symptoms and Remedies. Disponible en: http://www.imf.org/external/pubs/ft/weo/2016/02/index.htm?cmpid=Obanner\#ch2

Jackson, M. (2008). Social and Economic Networks. Disponible en: http://web.stanford.edu/ jacksonm/netbook.pdf

Kali, R., \& Reyes, J. (2010). Financial contagion on the international trade network. Economic Inquiry, 48(4), 1072 1101. doi: 10.1111/j.1465-7295.2009.00249.x

Kim, S., \& Shin, EH. (2002). A longitudinal analysis of globalization and regionalization in international trade: a social network approach. Social Forces, 81(2), 445-471. Disponible en: http://www.jstor.org/stable/3086478 https://doi. org/10.1353/sof.2003.0014

Lámbarry, Vilchis, F. (2016). Análisis estructural de la red económica de exportaciones e importaciones de la Alianza del Pacífico y el Mercado Común del Sur. Estudios Gerenciales, 32(141), 319-325. http://dx.doi.org/10.1016/j. estger.2016.10.004

Mahutga, M. (2006). The persistence of structural inequality? a network analysis of international trade, 1965-2000. Social Forces, 84(4), 1863-1889. https://doi.org/10.1353/sof.2006.0098

Meier, G. M. y Baldwin, R. E. (1957). Economic Development: Theory, History, Policy, New York: John Wiley \& Sons. DOI: https://doi.org/10.1017/S0022050700088860

Nemeth, R, y Smith, D. A. (1985). International Trade and World-System Structure: A Multiple Network Analysis. Review Fernand Braudel Center, 8(4), 517-560.

Newman, M. (2003). The structure and function of complex networks. SIAM Review, 45(2), 167-256.

Nieminen, J. (1974). On centrality in a graph. Scandinavian Journal of Psychology, 15, 332-336. DOI http://onlinelibrary.wiley.com/doi/10.1111/j.1467-9450.1974.tb00598.x/pdf

Nordlund, C. (2013). Seminario departamental. Core-periphery Structures: Operationalizing patterns of dependence and dominance in binary and valued networks. Disponible en: https://www.ceu.edu/event/2013-11-21/core-periphery-structures-operationalizingpatterns-dependence-and-dominance-binary

Organisation for Economic Cooperation and Development y World Trade Organization. (2015). Aid for Trade at a Glance 2015 Reducing Trade Costs for Inclusive, Sustainable Growth. Disponible en:http://www.keepeek.com/ Digital-AssetManagement/oecd/development/aid-for-trade-at-a-glance-2015_aid_glance-2015en\#.WA_SMtXhDX4\#page3

Organización Mundial del Comercio. (2016a). Acerca de la OMC. Disponible en: https://www.wto.org/spanish/thewto_s/whatis_s/who_we_are_s.htm

Organización Mundial del Comercio. (2016b). Trade Profiles. Disponible en: http://stat.wto.org/CountryProfile/WSDBCountryPFHome.aspx?Language=E Pemex. (2014). Anuario estadístico 2014. Disponible en: http://www.pemex.com/ri/Publicaciones/Anuario\%20Estadistico\%20Archivos/2014_ae_00_vc_e.pdf

Prebisch, R. (1950). The economic development of Latin America and its principal problems. New York: Cepal. Disponible en: http://archivo.cepal.org/pdfs/cdPrebisch/002.pdf 
Prebisch, R. (1964). Hacia una dinámica del desarrollo latinoamericano. México, D.F.: Fondo de Cultura

Económica. Disponible en: https://repositorio.cepal.org/bitstream/handle/11362/14230/S33898P922H_es.pdf?sequence $=1$

Prell, C., Sun, L., Feng, K., \& Myroniuk, TW. (2015). Inequalities in Global Trade: A CrossCountry Comparison of Trade Network Position, Economic Wealth, Pollution and Mortality. PLoS ONE, 10(12): e0144453. doi:10.1371/ journal.pone. 0144453

Reka, A., \& Barabási, A.L. (2002). Statistical mechanics of complex networks. Review of Modern Physics, 74(1), 47-97. https://doi.org/10.1103/RevModPhys.74.47

Reyes, J., Schiavo, S., y Fagiolo, G. (2008). Assessing the evolution of international economic integration using random-walk betweenness centrality: The cases of East Asia and Latin America. Advances in Complex Systems, 11(5), 685-702. https://doi.org/10.1142/S0219525908001945

Richardson, H.W. (1986). Economía regional y urbana. Madrid: Alianza.

Rombach, P., Porter, M.A., Fowler, J.H., y Mucha P.J. (2014). Core-Periphery Structure in Networks. SIAM Journal on Applied Mathematics, 74(1), 167-190, https://doi.org/10.1137/120881683

Rosales, O. (2015). Globalización, integración y comercio inclusivo en América Latina. Textos seleccionados 2010-2014. Disponible en: http://repositorio.cepal.org/bitstream/handle/11362/38952/S1500632_es.pdf

Secretaría de Economía. (2015). Comercio Exterior/Información Estadística y Arancelaria/Importaciones y exportaciones por país 1993-2016/Por socios. Disponible en: http://www.gob.mx/se/documentos/comercio-exterior-informacionestadistica-y-arancelaria-importaciones-y-exportaciones-por-pais-1993-2016-porsocios

Secretaría de Relaciones Exteriores, (2016). Información General de México. Disponible en: consulmex.sre.gob.mx/ guangzhou/index.php/zh/economia-y-comercio/mexico.

Serrano, M.A., y Boguñá, M. (2003). Topology of the World Trade Web. Physical Review E, 68 (015101). doi: https:// doi.org/10.1103/PhysRevE.68.015101

Serrano, M.A., Boguñá, M., y Vespignani, A.J. (2007). Patterns of dominant flows in the world trade web. Journal of Economic Interaction and Coordination, 2(2), 111-124. doi:10.1007/s11403-007-0026-y

Smith, D.A. y White, D.R. (1992). Structure and dynamics of the global economy: Network analysis of international trade 1965-1980. Social Forces, 70(4), 857-893. https://doi.org/10.2307/2580193

Steiber, S. (1979). The World System and World Trade: An Empirical Explanation of Conceptual Conflicts. The Sociological Quarterly, 20(1),23-36. https://doi.org/10.1111/j.1533-8525.1979.tb02182.x

The World Bank (2016). GDP Ranking. Disponible en: http://data.worldbank.org/datacatalog/gdp-ranking-table

United Nations Conference on Trade and Developmen. (2016). world investment report Investor Nationality: Policy Challenges 2016. Disponible en: http://unctad.org/en/PublicationsLibrary/wir2016_en.pdf

Uzzi, B., \& Spiro, J. (2005). Collaboration and Creativity: The Small World Problem1. American Journal of Sociology, 111(2), 447-504. https://doi.org/10.1086/432782

Watts, D. J., \& Strogatz, S. H. (1998). Collective Dynamics of “Small-World” Networks. Nature, 393, 440-442. https:// doi.org/10.1515/9781400841356.301

Wallerstein, I. (1979). The Capitalist World-Economy. United Kingdom: Cambridge University Press. Disponible en: http://catdir.loc.gov/catdir/samples/cam034/78002955.pdf

Wlather, O.J. (2015). Social Network Analysis and informal trade. SSRN Electronic Journal. doi:10.2139/ssrn.2593021

World Economic Forum (2016). Competitiveness Rankings. Disponible en:http://reports.weforum.org/ global-competitiveness-report-2015-2016/competitiveness-rankings/

World Trade Organisation. (2015a). Trade Profiles. Disponible en: http://stat.wto.org/CountryProfile/WSDBCountryPFView.aspx? Language $=\mathrm{E} \&$ Count ry $=\mathrm{MX}$

World Trade Organisation. (2015b). Trade Profiles. Disponible en: http://stat.wto.org/CountryProfile/WSDBCountryPFView.aspx?Language=E\&Count ry=E28 


\section{Anexo}

Tabla A1 Ejemplo de la Matriz de exportaciones-importaciones de los países de la OMC

Origen

Albania

Albania

Albania

Albania

Albania

Albania

Albania

Albania

Albania

Albania

Antigua and Barbuda Antigua and Barbuda Antigua and Barbuda Antigua and Barbuda Antigua and Barbuda Antigua and Barbuda Antigua and Barbuda Antigua and Barbuda Antigua and Barbuda Antigua and Barbuda

\section{Relación}

Exporta

Exporta

Exporta

Exporta

Exporta

Importa

Importa

Importa

Importa

Importa

Exporta

Exporta

Exporta

Exporta

Exporta

Importa

Importa

Importa

Importa

Importa
Destino

European Union (28)

76.7

Serbia

China

7.5

4.6

Turkey

3.7

FYR Macedonia

3.7

European Union (28)

64.2

China

6.8

Turkey

6.4

Serbia

4.1

United States of America

2.4

United States of America

27.1

European Union (28)

25.2

Curaçao

7.3

New Zealand $\quad 5.3$

Montserrat $\quad 3.0$

United States of America $\quad 52.3$

European Union (28) $\quad 10.4$

China

5.7

Trinidad and Tobago

Japan
4.5

3.0

Fuente: Elaboración propia a partir de Organización Mundial del Comercio. (2016b). Nota: *porcentaje de exportación/importación. 
Tabla A2 Red directa de México en el marco de la Organización Mundial del Comercio

\begin{tabular}{|c|c|c|c|c|c|c|c|c|}
\hline \multirow[t]{3}{*}{ Pais } & king 2 & & \multicolumn{2}{|c|}{ Ranking } & \multirow[t]{3}{*}{ Cercania } & \multirow[t]{3}{*}{ Intermediación } & \multirow[t]{3}{*}{ Cohesión } & \multirow[t]{3}{*}{ Grado } \\
\hline & \multirow{2}{*}{\multicolumn{2}{|c|}{ (PIB) }} & & & & & & \\
\hline & & & Exportación & Importación & & & & \\
\hline Unión Europea & $\mathrm{Na}$ & $16301833^{* * *}$ & 2 & 2 & 0.81415929 & 0.31928949 & & 287 \\
\hline China & 2 & 10866444 & 1 & 3 & 0.79310345 & 0.28700141 & 0.07147517 & 221 \\
\hline Estados Unidos & 1 & 17946996 & 2 & 1 & 0.70229008 & 0.16333292 & 0.07272001 & 204 \\
\hline Japón & 3 & 4123258 & 4 & 4 & 0.54599407 & 0.0289463 & 0.0862259 & 80 \\
\hline Brasil & 9 & 1774725 & 25 & 25 & 0.49329759 & 0.00506625 & 0.15793152 & 39 \\
\hline República de & 11 & 1377873 & 4 & 4 & 0.49197861 & 0.0042901 & 0.28307692 & 35 \\
\hline \multicolumn{9}{|l|}{ Corea } \\
\hline Canadá & 10 & 1550537 & 11 & 10 & 0.49066667 & 0.0035352 & 0.26461538 & 33 \\
\hline México & 15 & 1144331 & 13 & 12 & 0.47916667 & 0.00110394 & 0.26 & 26 \\
\hline Colombia & 38 & 292080 & 56 & 47 & 0.47179487 & 4.95E-04 & 0.47058824 & 18 \\
\hline Nicaragua & 123 & 12693 & 107 & 110 & 0.46938776 & 0.00358309 & 0.52564103 & 14 \\
\hline Belice & 170 & 1763 & 159 & 172 & 0.46819338 & 0.00372924 & 0.58181818 & 10 \\
\hline Guatemala & 71 & 63794 & 83 & 78 & 0.46819338 & $1.87 \mathrm{E}-04$ & 052777778 & 16 \\
\hline Cuba & 66 & 77150 & 112 & 83 & 0.46819338 & $1.41 \mathrm{E}-04$ & 0.77777778 & 10 \\
\hline Cabo Verde & 171 & 1630 & 185 & 182 & 0.46700508 & $1.00 \mathrm{E}-04$ & 0.69444444 & 10 \\
\hline Costa Rica & 79 & 51107 & 86 & 82 & 0.46700508 & $8.16 \mathrm{E}-05$ & 0.77777778 & 12 \\
\hline Panamá & 78 & 52132 & 80 & 75 & 0.46582278 & $1.66 \mathrm{E}-04$ & 0.78571429 & 15 \\
\hline Argentina & 21 & 548055 & 46 & 46 & 0.46582278 & $1.02 \mathrm{E}-04$ & 0.66666667 & 19 \\
\hline El Salvador & 103 & 25850 & 103 & 93 & 0.46582278 & $4.02 \mathrm{E}-05$ & 0.75 & 14 \\
\hline \multirow[t]{2}{*}{ Honduras } & 107 & 20152 & 92 & 91 & 0.46464646 & 0 & 0.89285714 & 12 \\
\hline & & Promedio: & & & 0.523818114 & 0.043220587 & & 57 \\
\hline
\end{tabular}

Fuente:elaboración a partir de The World Bank (2016) y World Trade Organisation, (2015b).

* Millones de dólares.

**Datos de 2015 de World Trade Organisation (2015b). 


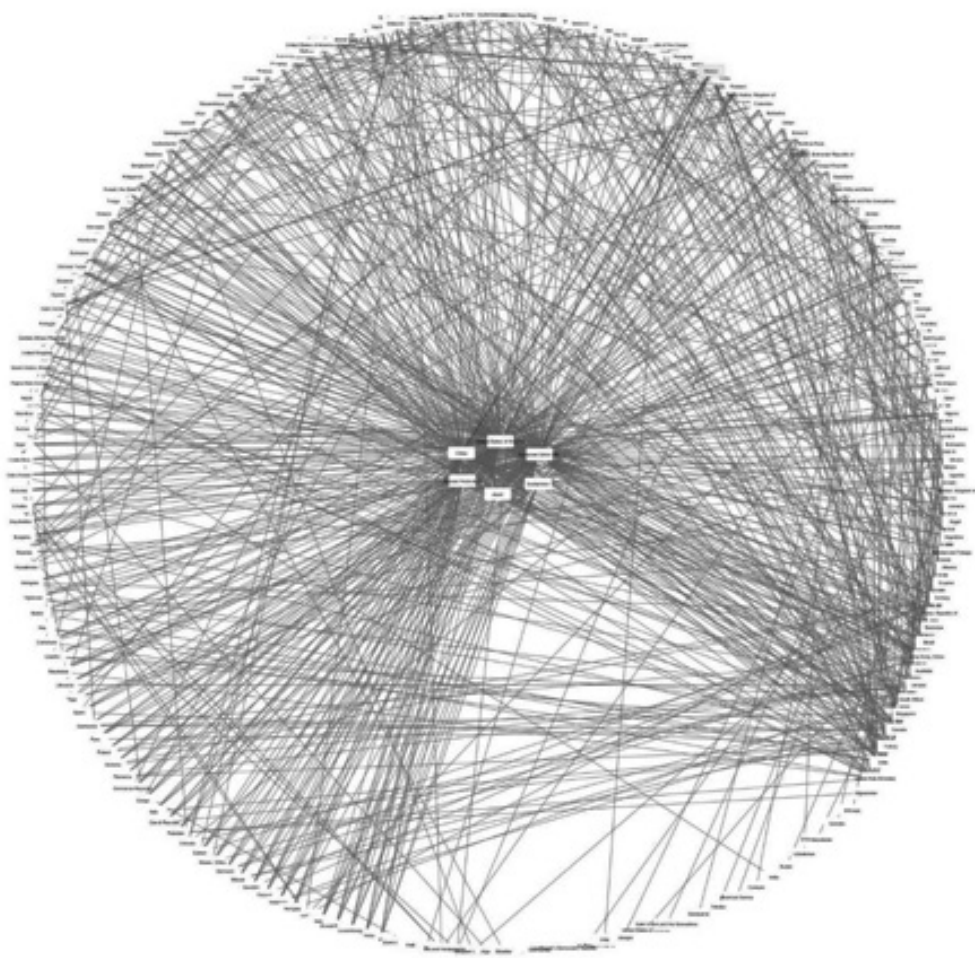

Figura 1. Red de exportaciones-importaciones de la OMC: México.

Fuente: elaboración propia con datos de la OMC y Cytoscape ver 3.3.0.

Nota: Los países en el núcleo son los que presentan los mayores coeficientes de centralidad. 